(2) Open Access Full Text Article

\title{
A mixed-methods approach to conducting Internal Revenue Service-compliant community health needs assessments: a case example for nonprofit hospital leaders
}

\author{
This article was published in the following Dove Press journal: \\ Journal of Healthcare Leadership \\ 29 October 2014 \\ Number of times this article has been viewed
}

\section{Willie H Oglesby \\ Ken Slenkovich}

Department of Health Policy and Management, College of Public Health, Kent State University, Kent, OH, USA
Correspondence: Willie H Oglesby Kent State University, College of Public Health, PO Box 5190, 750 Hilltop Drive, Kent, $\mathrm{OH} 44242$, USA

Tel +l 3307327825

Fax +I 8663302654

Email woglesby@kent.edu
Background: The Patient Protection and Affordable Care Act created new requirements for nonprofit hospitals to conduct a Community Health Needs Assessment (CHNA) at least once every 3 years, with a significant tax penalty for noncompliance. While some resources exist to help nonprofit hospital leaders conduct various aspects of a CHNA, few reflect the new Internal Revenue Service requirements.

Methods: Many different models of CHNAs have emerged over the years. Although each has its unique features, the essential elements of a CHNA include engaging stakeholders, defining the community, gathering sufficient representative data, prioritizing information, and reporting results. In this paper, we expand upon this basic approach by offering a practical step-by-step guide to conducting CHNAs that meets new Internal Revenue Service regulations.

Results: We developed and tested this methodology in partnership with several nonprofit hospital systems in Northeast Ohio, USA. In this paper, we discuss our use of the methodology and identify recommendations for other nonprofit hospital leaders.

Conclusion: The methodology presented in this paper is a cost-effective approach to satisfying new CHNA requirements and nonprofit hospital leaders should consider using it or modifying it to fit their unique needs.

Keywords: Affordable Care Act, CHNA, community benefit, community hospital

\section{Background}

Community Health Needs Assessments (CHNAs) have been conducted by public health agencies for decades and are widely regarded as an important prerequisite for planning effective community-based programs and services. Many different models of CHNAs have emerged over the years, including the Community Health Assessment and Group Evaluation (CHANGE) model, ${ }^{1}$ Mobilizing for Action through Planning and Partnerships (MAPP), ${ }^{2}$ Planned Approach To Community Health (PATCH) model, ${ }^{3}$ PRECEDE-PROCEED model, ${ }^{4}$ Community Diagnosis, ${ }^{5}$ and others. Although each model has its unique features, the essential elements of a CHNA include engaging stakeholders, defining the community, gathering sufficient representative data, prioritizing information, reporting results, and developing and evaluating a community health improvement plan.

Although CHNAs have traditionally been conducted by public health agencies, they have also been conducted by other local community institutions, including nonprofit community hospitals. CHNAs have been useful tools for nonprofit hospitals to help plan and prioritize their community benefit programs. Since 1969, the Internal Revenue Service (IRS) has granted nonprofit hospitals tax exemption status in exchange for 
providing community benefits. ${ }^{6}$ These community benefits include free or discounted medical care for people who cannot afford it, Medicaid costs, community health improvement programs, health professions education, research, and other qualifying expenditures. ${ }^{7}$

In March 2010, the Patient Protection and Affordable Care Act created new requirements of nonprofit hospitals including conducting a CHNA and adopting an implementation strategy at least once every 3 years starting with taxable years beginning after March 23, 2012. ${ }^{8}$ A subsequent notice issued in 2011 (Notice 2011-52) defined requirements of CHNAs including a description of the community served by the hospital facility; the process used to conduct the assessment, including how the hospital took into account input from community members and public health experts; identification of any persons with whom the hospital has worked on the assessment; and the health needs identified through the assessment process including other specific technical requirements. ${ }^{9}$ Failure to properly conduct a CHNA could result in an excise tax of $\$ 50,000$ per facility per year. ${ }^{10}$

While some resources exist to help nonprofit hospital leaders with various aspects of CHNAs, few discuss how to conduct them in response to the new IRS regulations. This leaves nonprofit hospital leaders with little information on how to successfully conduct CHNAs in response to new IRS regulations, exposing their institutions to significant penalties for noncompliance. To address this gap, we offer a practical approach to conducting IRS-compliant CHNAs based on our work with several nonprofit hospital systems in Northeast Ohio, USA. The methodology described here can be used with standalone hospitals or multihospital systems. We conclude with a discussion on the lessons learned from our experiences and offer recommendations. The information is presented prospectively to help other hospital leaders conduct their own CHNA using this methodology.

\section{Methods}

A stepwise approach should be taken to conduct the CHNA, which includes engaging stakeholders, defining the community, gathering sufficient representative data, prioritizing information, and reporting results.

\section{Engaging stakeholders}

CHNAs should be conducted in collaboration with stakeholders, who are groups of people that can affect, and be affected by, the outcomes of CHNAs. In nonprofit hospitals, this includes the organization's tax accountant, who can advise on changes to IRS regulations pertaining to the CHNA and who will submit it as a part of the nonprofit hospitals annual information return; the community benefit manager, who is aware of existing community health programs and can connect with other community organizations; senior leadership, who can maintain institutional support and link the CHNA to the institution's strategic plans; service line/departmental leaders, who can provide detailed information on different patient groups; providers of patient care, who can contribute their firsthand experiences; patients and families who consume services; and other people inside the hospital who can affect, or can be affected by, a CHNA. CHNAs should also include people outside of the hospital, including community organizations, community leaders, and community residents. Community organizations and leaders should include governmental and nonprofit health and human service organizations; health departments; planning boards; academic institutions; faith-based organizations; youth service groups; environmental groups; and other community organizations that have a stake in the health and wellbeing of the community. Lastly, community residents themselves should be included in the CHNA. This should include people who have had experience with the hospital and those who have not. All of these stakeholders should comprise a committee that will lead the development and implementation of the CHNA.

\section{Defining the community}

Most hospitals serve patients and families across a wide geographic region, particularly if the hospitals offer specialty services not widely available in the region or if they are highly ranked in a particular field of medicine. Additionally, since many nonprofit hospitals have a mission to serve all patients regardless of ability to pay, these hospitals can attract patients over long distances.

Adding every area where every patient resides to the hospital's "community" is unrealistic, since in some areas the hospital many only serve a small number of residents. In addition, using such a large geographic area as "the community" for the purposes of the CHNA sets expectations that the hospital will do something to address any health needs identified in those outlying areas. Lastly, spreading limited resources across a wide geographic area can dilute the impact of community benefit programs.

To identify the community served by a hospital, the zip codes of patients from hospital discharge and patient visit records during the last calendar year can be drawn on population density maps to identify high-concentration areas. In addition, internal market analyses and expansion plans can be consulted to determine the hospital's future geographic 
service area. Together, the patient-density maps, marketing analyses, and expansion plans will provide an important snapshot of the hospital's current patient footprint as well as the hospital's new service areas, which can be used to decide upon the community served by the hospital for the CHNA.

Depending on its size, a hospital's community may encompass many neighborhoods, several cities or towns, or span multiple counties. The larger the community served by the hospital, the more diverse it will likely be. Different areas in the community will likely have unique health outcomes and risk factors as well as varied perceptions of community health problems and solutions. Hospital leaders should design CHNAs in a way that accurately accounts for these differences across the hospital's community.

After the hospital's community is identified, a decision should be made to clarify the community's geographic boundaries. The geographic boundaries of a community can be expressed in a variety of levels, including census tracts, zip codes, neighborhoods, municipal areas (eg, cities, villages, townships), counties, multicounty regions, or other similar geographic subdivisions. Each level of geographic subdivision has different advantages and disadvantages for a CHNA. Lower-level geographic subdivisions, such as census tracts or neighborhoods, can more precisely define a hospital's community, but health data at those levels are usually unavailable. Higher-level geographic subdivisions, such as counties and multicounty regions, usually have more readily available information, but they can mask variations within the geographic subdivision. Unless the hospital has access to representative data across the entire community (not just from their own electronic medical records, which only include their patients), hospitals should consider expressing the community's geographic boundaries at the county level, since epidemiologic data is most available at that level.

\section{Gathering sufficient representative data}

To be effective, CHNAs require an adequate amount of information across the community. Data used in CHNAs need to be representative of the community served and must address the broad health issues and contextual factors experienced by the community. This can be accomplished using a mixed methods approach that includes gathering epidemiologic and contextual data, interviewing community leaders, and conducting focus groups with community residents.

\section{Epidemiologic and contextual data}

Epidemiologic and contextual data can be collected from national sources, such as County Health Rankings,
Community Health Status Indicators, Community Health Needs Assessment Toolkit, and the Annie E Casey Foundation. These web sites report data at the county level, which underscores the reason for defining the hospital's community at this level. Most of the data on these sites are drawn from federal sources such as the National Vital Statistics System, Behavioral Risk Factor Surveillance Survey, Youth Risk Behavior Survey, Substance Abuse and Mental Health Services Administration, National Notifiable Diseases Surveillance System, Environmental Protection Agency, Centers for Medicare and Medicaid Services, National Center for Education Statistics, Uniform Crime Reports, Department of Agriculture, National Environmental Public Health Tracking Network, and others, creating a comprehensive array of indicators. Demographic data can be collected from the US Census Bureau using American FactFinder and can also be obtained at the county level.

Although these national sources of data cover a wide variety of health areas, they are limited in depth. To supplement the data retrieved from national sources, data should also be collected from state and local health departments; the department of education; state and local offices that administer government assistance programs (including Medicaid, food/nutrition assistance, housing, employment, disability, child care assistance, etc); state and local drug addiction and mental health boards; state and local hospital associations; local/regional health registries; and other state and local sources. Often, the health needs of children are different than adults so health data should be collected for adults and children.

To help distill the large amount of health data that will be obtained, a systematic prioritization process will need to be undertaken (explained below). To aid in this prioritization process, data should be collected for each county in the hospital's community, the state, and the nation. In addition, data from two demographically similar peer counties should also be collected for each county in the hospitals' community. Lastly, Healthy People 2020 targets should be obtained for any health area represented in the epidemiologic data. All of these data points will be used to prioritize the health needs in the communities served by the hospital (explained below).

\section{Community leader interviews}

CHNAs should not rely solely on epidemiologic, demographic, and other quantitative data. Nor should they be absent of the perspective of the communities they are assessing. To gain additional insight into the health status of the communities served by the hospital, community leaders 
and community residents should be consulted. To solicit the depth of information that community leaders can provide, a qualitative approach should be taken. Due to barriers related to scheduling with community leaders, interviews are preferable to focus groups.

Care should be taken to identify a set of community leaders who represent the broad interests of the communities served by the hospital. The IRS specifically identifies the medically underserved, low-income persons, minority groups, those with chronic disease needs, and leaders from local public health agencies and departments who have special knowledge and expertise in public health. ${ }^{9}$ At a minimum, community leaders should be consulted to ascertain what they think are the significant health needs of children and adults in their communities. It is advisable to also get their opinion on the factors that affect those health needs, other existing community health needs assessments that they may be aware of, possible collaboration opportunities with the hospital to address the health needs, suggestions on what they think the hospital can do to address the significant health needs in the community, and other areas that can help inform the CHNA.

The interviews can be conducted over the telephone or in-person. Representative notes of the interviews should be taken during the interview. Although it is not necessary to transcribe the interview verbatim, it is helpful to document interesting, unique, and representative quotes. At the conclusion of the interview, the interviewee should be asked for his/her permission to be listed in the CHNA, as it is also a requirement in the regulations. ${ }^{9}$ After all of the interviews have been conducted, a content analysis should be conducted on the interview notes to identify emergent themes and health areas.

\section{Community resident focus groups}

Community residents should also be engaged in CHNAs to identify what they think are the significant health needs of children and adults in their communities, the factors that affect those health needs, other existing community health needs assessments, possible collaboration opportunities, and to get suggestions on what the hospital can do to address the significant health needs in the community. There are several ways to engage community members, including administering surveys or conducting interviews or focus groups.

If resources permit, a population-based survey containing valid and reliable measures with a stratified sample of representative community residents and appropriate weights is preferred, but this method can be cost-prohibitive due to the expertise needed to construct, administer, analyze, and interpret the results of the survey. Individual interviews can also be conducted, but given the size of many hospitals' service areas, this approach can be labor intensive and time consuming. When conducted in conjunction with populationbased data, such as epidemiologic and contextual data described above, focus groups can be a very useful tool to engage community residents in CHNAs.

For CHNAs, focus groups should consist of eight to 12 people and meetings should last no longer than 60 minutes so that all members have a chance to contribute but do not become fatigued by a lengthy conversation. For consistency, the questions asked of community residents should be similar to those asked of community leaders. The composition of the focus groups can either be random (based on participants' availability for the predetermined date, time, and location) or can be purposive (based on some attribute such as a participant's race, age, sex, etc). In general, purposive sampling should be reserved only for CHNA projects where it is important to explore differences between certain groups because the logistics and analysis involved are more complex. For CHNAs that seek to gain a broad community perspective, it is acceptable to convene multiple focus groups comprised of a diverse mix of participants.

Regardless of the sampling strategy used, care should be taken to convene focus groups on days, times, and locations that are convenient to all segments of the community. This includes morning, afternoon, and weekend sessions at senior centers, shopping malls, work readiness centers, faith organizations, youth service organizations, and other locations. When possible, locations should be convenient to public transportation hubs for residents who rely on such services. In addition, financial incentives should be provided to compensate participants for their time and expense and to encourage their participation. Lastly, a brief demographic questionnaire should be used to describe focus-group participants. Questions should include age, sex, race, ethnicity, zip code of residence, years lived in the community, healthinsurance status and source, number of children in the home, and if someone in the home currently has any chronic conditions. Additional questions may be added, but should be used sparingly. The purpose of the demographic questionnaire is to gather basic information about focus-group participants so that it can be compared to the epidemiologic and contextual data discussed above to determine if focusgroup participants adequately represented the community. It is not a substitute for a population-based survey. 
As with the community-leader interviews, it is not necessary to transcribe verbatim the conversations of each focus group. However, interesting, unique, and representative quotes should be documented. This can be accomplished by having a note taker sitting in the back of the room. After all of the focus groups have been completed, a content analysis should be conducted on the notes to identify emergent themes and health areas.

\section{Other community health needs assessments}

CHNAs are routinely conducted by local public health groups as a part of evidence-based public health practice and are frequently required by their funders. These include groups that provide $\mathrm{HIV} /$ sexually transmitted disease prevention and treatment services, family planning services, tobacco cessation programs, environmental impact assessments, rape prevention programs, violence and injury prevention, substance abuse prevention and treatment services, maternal and child block grant initiatives, and others. Although the focus and methods will likely be different, CHNAs conducted by other public health groups will likely yield additional insight on a community's health and therefore should be incorporated in the process to identify the top health needs of a community.

\section{Prioritizing information}

Gathering representative data for $\mathrm{CHNAs}$ will produce a large amount of information that can be difficult to summarize. In addition, because the purpose of the CHNA is to arrive at a list of prioritized health needs for a community, a systematic way to combine the quantitative data (ie, epidemiologic data) and qualitative data (ie, input from community leaders and residents) is needed.

\section{Summarizing epidemiologic data: the relative ranking method}

There are a variety of ways to summarize large amounts of quantitative health data for CHNAs. One of the most user-friendly is the relative ranking framework. ${ }^{11}$ In this framework, the value for each county's health indicator is compared to the values of two demographically similar peer counties, the state, the nation, and the Healthy People 2020 target, if one was available, and then plotted on a matrix (see Figure 1).

For example, if the diabetes diagnosis rate in Allan County was higher than its two demographically similar peer counties, the state, the nation, and the Healthy People 2020 target, then it would be placed in the upper-left-hand

\section{Allan County adults}

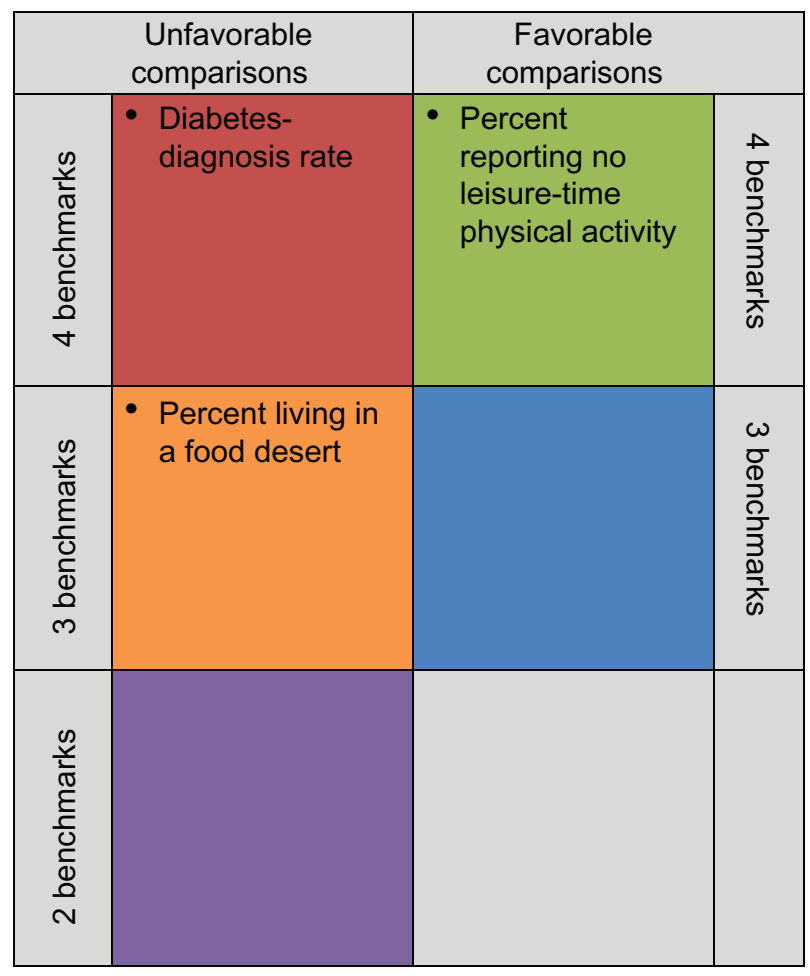

Figure I Prioritization matrix example.

side of the matrix (shaded in red). Similarly, if the percent of people living in a food desert in Allan County is higher than its two demographically similar peer counties, the state, and the nation, but there is not a Healthy People 2020 target, it would be placed in the orange shaded area in the middle of the left-hand side. Lastly, if the percent of adults reporting no leisure-time physical activity in Allan County was lower in Allan County than all other comparators, it would be placed in the upper-right-hand side of the matrix (shaded in green). This process would continue until all of the health indicators for adults and children were plotted on matrices for all of the counties in the hospital's service area. When completed, you should have two matrices for each county in the hospital's community (one for adults and one for children).

This relative ranking approach is one of the most userfriendly methods for summarizing large amounts of quantitative health data because it results in a series of matrices that graphically highlight the greatest health needs in a community. Indicators are rank-ordered using a multiplecomparison method, which allows hospital leaders to quickly identify problem areas for improvement. The graphic representation of the data is also useful for public presentations and other general audiences who may have limited experience working with health-related quantitative data. 


\section{Adding community leader and resident input}

After the matrices have been created, the list of top health needs identified by community leaders and residents should be consulted. Frequently, the top health needs identified by the community are similar to what is already represented in the epidemiologic data, which is a good reinforcement of the data. However, there will be occasions where community leaders and residents identify health needs that are not contained in the matrices. In some cases, this will be due to a complete lack of epidemiologic information on the particular health area, which is common in some health areas (ie, substance abuse and mental health) and particularly so for children. In other cases, it will stem from differences between the overall community (as represented in the epidemiologic data) and the subgroups of residents and community leaders that participate in the CHNA. Regardless of the reason, when differences arise between the results of the epidemiologic analysis and the input from community leaders and residents, careful deliberation must be taken. As a general rule in CHNAs, if there is consensus among community leaders and community residents that a particular health area should be considered a top community health need, it should be included as such.

\section{Reporting results}

IRS regulations require that CHNAs conducted by nonprofit hospitals "be made widely available to the public", which can be satisfied by posting it on the hospital's web site by the required posting date (see Page 17 of IRS Notice 2011-52 for a more detailed explanation of how to meet this requirement without a web site). ${ }^{9}$ Because CHNAs are intended to be public documents, they should be written in a manner that is easily understandable by different segments of the community, including those with low levels of educational attainment. This means that CHNAs should use plain language (avoid jargon); use tables, graphs, and charts to illustrate complex or dense information; and include a succinct executive summary.

\section{Results}

In this article, we propose a method for conducting CHNAs that is compliant with IRS regulations for standalone nonprofit hospitals and multihospital systems, equipping hospital leaders with a practical step-by-step guide to help them meet this new requirement. Our experience conducting IRScompliant CHNAs is based on our work with several large nonprofit hospital systems in Northeast Ohio that include collaborative multisystem CHNAs and standalone CHNAs.

\section{Engaging stakeholders}

In our CHNA projects, we worked with hospitals and hospital systems that used multilevel governance structures to engage stakeholders in different ways. Some formed internal teams consisting of key administrative and community benefit staff, providers, and service line managers. Some supplemented their internal teams with input from external stakeholders who served on the hospital's community benefit advisory committee. Across the hospitals and systems, there were differences in terms of the level of internal staff who were engaged in the CHNA process with some hospitals including senior level administrators and providers, and others including primarily mid-level staff. Although each hospital and system approached stakeholder engagement in different ways, each included people who have a stake in the process and outcomes of the CHNA. This included the organization's tax accountant, community benefit managers, senior administrators, and care providers. Some also included board representatives and community members.

\section{Defining the community}

Like many nonprofit hospitals, the hospitals and systems we work with serve patients and families from across a wide geographic region. To identify its "community" for the purposes of the CHNA, one hospital system exclusively used population density maps of prior-year discharges to identify areas where at least $75 \%$ of patients reside. Others supplemented their discharge analysis by examining the geographic footprint of the entire hospital system, which allowed them to include areas with newer and smaller facilities (with relatively fewer patient discharges) and ambulatory care facilities (which would be missing from the discharge analysis). Based on the availability of health information at the national and local levels, all of the hospitals and systems we worked with choose to define their community at the county-level. Some define their community as one county and others define it as multiple counties.

\section{Gathering sufficient representative data}

Data for all of the CHNAs came from the sources listed above. Although these data sources have many advantages, they also have limitations. For example, when we collected data from the County Health Rankings dataset, the adult-obesity rate was from the 2010 Behavioral Risk Factor Surveillance Survey, the rate of preventable hospital stays was from the 2011 Dartmouth Atlas of Health Care, and the mental health provider ratio was from the 2013 National Provider Identifier Registry - all in different years. In addition, these sources did not include data on all community health issues or popula- 
tions; most of the data focused on adults, with substantially less available for children, and the data were reported in the aggregate and not stratified by race/ethnicity, sex, or other important differences. In spite of these limitations, however, these databases are currently the best comprehensive source of epidemiologic information for CHNAs.

Interviews with community leaders lasted approximately 30 minutes in length, which seemed to be the optimal time period for this group. No incentives were provided to community leaders for participating in the CHNAs and this was not observed as a barrier to participation. The initial list of community leaders was prepared by the hospitals and additions were made when the community leaders identified other leaders who should be interviewed.

Focus groups with community residents lasted approximately 45-60 minutes in length, depending upon the size of the group. During the interviews, we asked community leaders to advise us on which locations and advertising strategies would be best to recruit a representative sample of community residents. We ended up holding focus groups at an Urban League office, public libraries, the regional campus of a university, a grocery store, and health departments during work hours, evenings, and on weekends. We required preregistration by calling, texting, or emailing to ensure that we kept the groups to a maximum of 15 and that we brought enough materials. Focus-group participants were provided a \$50 VISA gift card for participating in the focus group. Due to Institutional Review Board considerations, we only recruited adults aged 18 years or older in the focus groups.

The data collected from community leaders and community residents were a very valuable contribution to the CHNAs for several reasons. Firstly, they confirmed many of the findings from the epidemiologic data, particularly around chronic diseases such as heart disease and diabetes, which was an important validation of the quantitative data. Secondly, they identified important health areas that were not represented in the epidemiologic data, such as specific mental and behavioral health issues and a myriad of child health issues, because those population-level measures are largely not available at the county level. Lastly, they identified practical opportunities for the hospitals to help address the identified health needs, including collaboration opportunities with other community organizations and resident groups.

During the data-collection process, several important data gaps were identified. As discussed, some of the most striking gaps were in mental and behavioral health and the overall significant gap in child health data, compared to what is available for adults. These data do exist at the national, and to some degree, state levels, but they are largely missing at the county level. Of what is available, the data are usually counts or other metrics of service utilization, which are not representative of the larger population, but only of those that use the services. To address this gap in future CHNAs, the hospitals and systems are evaluating various strategies to increase this information, including partnering with health departments to oversample and add questions to the adultbased Behavioral Risk Factor Surveillance Survey and the child-based Youth Risk Behavior Survey, although this will require more financial resources and technical expertise.

\section{Prioritizing information}

Epidemiologic data were collected for each of the selected indicators for the target counties, two peer counties for each target county, the State of Ohio, and the nation. When available, the Healthy People 2020 target was also included. We compared each data point for each county using the methods described above. We did this separately for adult data and child data. This produced two matrices (one adult and one child) for each county in each hospital's community.

After all the matrices were created, the results of the community leader interviews and community resident focus groups were summarized, and other existing CHNAs were collected; a group facilitation process was conducted to identify which health areas would become the "prioritized health needs" for the purposes of the CHNAs. The group was comprised of the stakeholders described above. We scheduled a 2-hour time block to reserve enough time to carefully review the epidemiologic data, the input from the community leaders and community residents, and the results of other CHNAs. Disagreements about what should be considered a prioritized health need were fully discussed so that the final list of prioritized health needs represented a consensus among participating stakeholders. A series of votes were cast to ensure equitable contribution from each member.

\section{Reporting results}

The CHNA process and outcomes were summarized in two documents: a summary and a detailed data appendix. Both documents presented information in a user-friendly format, used plain English language, and avoided technical jargon, when possible. Charts and graphs were used to illustrate quantitative information and paragraphs were used to interpret the contents of the tables, charts, and graphs. Both documents were edited by the stakeholders mentioned above. The final documents were submitted to each hospital system's board of directors for approval. After approval, 
the documents were converted to PDFs and posted on the hospital system's web site. A press release was drafted for each hospital system and submitted to local media outlets, several of which covered the story. The CHNAs were also used by several departments and centers within the hospital systems to help satisfy the needs-assessment requirements of their various accrediting and certifying agencies.

\section{Conclusion}

This paper presents a practical step-by-step process to help nonprofit hospital leaders conduct CHNAs that are compliant with new IRS regulations created by the Patient Protection and Affordable Care Act. The methodology described here can be used by standalone hospitals and multihospital systems. We developed and tested this methodology in partnership with several nonprofit hospital systems in Northeast Ohio. The CHNAs that resulted from this approach successfully engaged stakeholders, accurately captured representative data, incorporated input from community leaders and residents, identified data gaps to fill, and facilitated consensus on prioritized health needs. The final reports generated positive press attention and are being used to develop implementation strategies that address the identified health needs in partnership with other hospitals and community groups. In summary, this methodology was a cost-effective approach to satisfying these new CHNA requirements and nonprofit hospital leaders should consider using this approach or modifying it for their own unique needs.

\section{Acknowledgments}

The authors gratefully acknowledge the productive partnerships with collaborating nonprofit hospitals and the community leaders and community residents who participated in the community health needs assessments we conducted.

\section{Author contributions}

All authors made substantial contributions to conception and design, acquisition of data, or analysis and interpretation of data; took part in either drafting the article or revising it critically for important intellectual content; gave final approval of the version to be published; and agree to be accountable for all aspects of the work in ensuring that questions related to the accuracy or integrity of any part of the work are appropriately investigated and resolved.

\section{Disclosure}

The authors received contracts from several nonprofit hospitals to conduct the community health needs assessments described in this manuscript. The authors report no other conflicts of interest in this work.

\section{References}

1. Community health assessment and group evaluation (CHANGE): Building a foundation of knowledge to prioritize community needs [webpage on the Internet]. Atlanta: Centers for Disease Control and Prevention; 2012 [updated October 25, 2013; cited April 13, 2014]. Available from: http:// www.cdc.gov/nccdphp/dch/programs/healthycommunitiesprogram/ tools/change.htm. Accessed October 2, 2014.

2. Mobilizing for action through planning and partnerships (MAPP) [webpage on the Internet]. Washington: National Association of County and City Health Officials [cited April 13, 2014]. Available from: http://www. naccho.org/topics/infrastructure/Mapp/index.cfm. Accessed October 2, 2014.

3. Kreuter MW. PATCH: Its origin, basic concepts, and links to contemporary public health policy. Journal of Health Education. 1992;23(3):135-139.

4. Green LW, Kreuter MW. Health Program Planning: An Educational and Ecological Approach. 4th ed. New York: McGraw-Hill; 2005.

5. Haglund BJ. Community diagnosis. A theoretical model for prevention in primary health care. Scand J Prim Health Care. 1983;1(1):12-19.

6. Colombo J, Griffith G, King J. Charity Care for Nonprofit Hospitals: A Legal and Administrative Guide. New York: Aspen Publishers; 2009.

7. Singh SR. Community benefit in exchange for non-profit hospital tax exemption: current trends and future outlook. J Health Care Finance. 2013;39(3):32-41.

8. New requirements for 501(c)(3) hospitals under the Affordable Care Act [webpage on the Internet]. Washington: Internal Revenue Service [updated March 4, 2014; cited April 13, 2014]. Available from: http:// www.irs.gov/Charities-\&-Non-Profits/Charitable-Organizations/NewRequirements-for-501(c)(3)-Hospitals-Under-the-Affordable-Care-Act. Accessed October 2, 2014.

9. Preston Quesenberry. Notice and Request for Comments Regarding the Community Health Needs Assessment Requirements for Tax-Exempt Hospitals (Notice 2011-52). Washington: Internal Revenue Service. Available from: http://www.irs.gov/pub/irs-drop/n-11-52.pdf. Accessed April 13, 2014.

10. Requirement of a Section 4959 Excise Tax Return and Time for Filing the Return; Final and Temporary Regulations. 78 Federal Register 158: August 15, 2013:49681-49682.

11. Studnicki J, Steverson B, Myers B, Hevner AR, Berndt DJ. A community health report card: comprehensive assessment for tracking community health (CATCH). Best Pract Benchmarking Healthc. 1997;2(5):196-207.
Journal of Healthcare Leadership

\section{Publish your work in this journal}

The Journal of Healthcare Leadership is an international, peer-reviewed, open access journal focusing on leadership for the health profession. The journal is committed to the rapid publication of research focusing on but not limited to: Healthcare policy and law; Theoretical and practical aspects healthcare delivery; Interactions between healthcare and society and evidence-based practices;

\section{Dovepress}

Interdisciplinary decision-making; Philosophical and ethical issues; Hazard management; Research and opinion for health leadership; Leadership assessment. The manuscript management system is completely online and includes a very quick and fair peer-review system. Visit http://www.dovepress.com/ testimonials.php to read real quotes from published authors. 